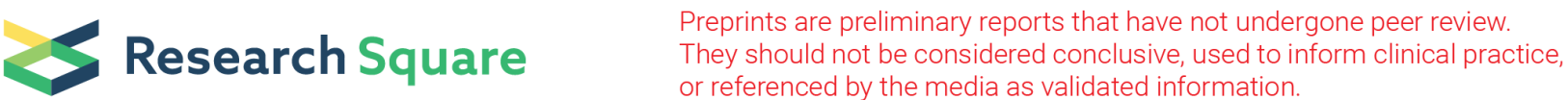

\section{All-Trans Retinoic Acid Modulates The Immune Status of M2 Macrophages in Experimental Periodontitis Induced by Porphyromonas Gingivalis in Mice}

\section{Wenjuan Zhang ( $\sim$ 18941607957@163.com )}

Second Affiliated Hospital of Jinzhou Medical University https://orcid.org/0000-0002-3344-5909

Ning Guan

First Affiliated Hospital of Jinzhou Medical University

\section{Xiu-qiu Gao}

2nd Affiliated Hospital of JMU: Second Affiliated Hospital of Jinzhou Medical University

\section{Lin-yuan Wang}

Second Affiliated Hospital of Jinzhou Medical University

\section{Research Article}

Keywords: Chronic periodontitis, all-trans retinoic acid (ATRA), M2 macrophage. cytokine, inflammation

Posted Date: December 13th, 2021

DOI: https://doi.org/10.21203/rs.3.rs-1142636/v1

License: (c) (i) This work is licensed under a Creative Commons Attribution 4.0 International License.

Read Full License 


\section{Abstract}

Background: M2 macrophages are important innate immune cells that participate in the pathogenesis of periodontitis. The effect of all-trans retinoic acid (ATRA) on the immune status of M2 macrophages in periodontitis has not been reported.

Methods: An experimental model of periodontitis was established in mice by oral administration of Porphyromonas gingivalis, and then ATRA or vehicle was administered orally to model mice every other day (P.g+ATRA and P.g+CMC mice, respectively). Flow cytometry was used to analyze the numbers of F4/80+CD206+ M2 macrophages in the gingiva, spleen, and peritoneal lavage fluid (PLF). M2 macrophage-related cytokines were quantified by real-time reverse transcription-polymerase chain reaction.

Results: Compared with P.g+CMC mice, P.g+ATRA mice showed a significantly reduced cemento-enamel junction to alveolar bone crest (CEJ-ABC) distance. The percentage of F4/80+CD206+ M2 macrophages in gingiva, PLF and spleen in model mice increased after ATRA treatment. The mRNA expression levels of M2 macrophage-related cytokines (IL-10, TGF-b1 and Arg-1) in gingiva, PLF and spleen of P.g+ATRA mice were higher than those of $P . g+C M C$ mice.

Conclusions: These results suggested that ATRA modulates the immune status of M2 macrophages and provides protection against periodontitis by enhancing $\mathrm{M} 2$ activation.

\section{Introduction}

Periodontitis is a disease involving the destruction of periodontal supporting tissue caused by unattached subgingival plaque and its metabolites in the gingival sulcus. Periodontitis is characterized by the loss of attachment, alveolar bone resorption, and periodontal pocket formation. The host immune response, including both innate and adaptive immune responses, is initiated against periodontal pathogens and plays an important role in the occurrence, development, and prognosis of periodontitis[1]. Resident macrophages in gingival tissue secrete large numbers of proinflammatory cytokines and chemokines after interactions with periodontal microorganisms. The macrophages participate in the phagocytosis and inflammation of periodontal pathogenic microorganisms and regulate alveolar bone resorption [2].

Macrophages are heterogeneous cells with strong plasticity and functional diversity that play key roles in both innate and adaptive immunity. Macrophages can be divided into many types based on their activated phenotypes and functions, including the classically and alternatively activated macrophages, designated as M1 and M2 macrophages, respectively. The phenotypic variations in macrophages induced by activation are reversible. M2 macrophages are activated by Th2 cytokines, such as interleukin (IL)-4, IL-13, and IL-10 [3,4], and secrete anti-inflammatory cytokines such as IL-10 and IL-1 receptor antagonists, as well as high levels of mannose receptor (CD206), arginase-1 (Arg-1), chitinase-3-like protein 1 (CHI3L1), and resistin-like molecule alpha (RELMa). M2 macrophages play important roles in the repair and remodeling of tissue damage caused by pathogenic microorganisms in the later stages of 
inflammation [5,6]. Intraperitoneal injection of M2 macrophages was reported to reduce experimental colitis in mice via an IL-10-dependent mechanism [7]. In a study in an experimental periodontitis model, adiponectin was suggested to participate in M2 macrophage polarization and host response by regulating the JMJD3-IRF4 axis, thus alleviating alveolar bone resorption [8]. Therefore, regulation of macrophage polarization to M2 macrophages may represent a new strategy for the treatment of periodontitis.

All-trans retinoic acid (ATRA) is an active metabolite of vitamin A that has a wide range of immunomodulatory effects and plays important roles in immune cell differentiation and activation as well as maintenance of the immune system. Studies showed that ATRA can be used in the treatment of a wide range of autoimmune diseases [9]. ATRA also regulates dendritic cells and reduces their antigen presentation ability, which further stimulate the T cells to respond to Th2 [10]. In addition, the combination of ATRA and IL-4 significantly increased the expression of arginase-1 (Arg-1), which is a marker of M2 activation [11]. However, there have been no studies on the effects of ATRA on the immune status of M2 macrophages in periodontitis. In this study, we examined the effects of ATRA on the immune status of M2 macrophages in periodontitis using a mouse model of periodontitis.

\section{Materials And Methods}

\section{Mice}

The experiments were performed in 7-week-old specific pathogen free C57BL/ 6 female mice weighing $\sim 18 \mathrm{~g}$, with intact dentition and no caries or periodontal disease. Mice were obtained from the Animal Center of Jinzhou Medical University. Animals were kept in individual cages with ad libitum access to food and water and fed adaptively for 1 week before establishment of the periodontitis model. The experimental procedures and animal treatment protocols were approved by the Ethics Committee of Jinzhou Medical University.

\section{Mouse periodontitis model}

Porphyromonas gingivalis W83 was cultivated on brain heart infusion (BHI) blood agar medium (Solarbio, Beijing, China) (containing 5\% sheep's blood (Solarbio), 1\% hemin (Sigma-Aldrich, MO, USA), and $0.1 \%$ vitamin K1 (Huayang, Jiangsu, China)) for 5-7 days. Colonies were scraped off the plates and re-suspended in BHI liquid culture medium. P. gingivalis W83 was collected after $24 \mathrm{~h}$. The bacterial density was adjusted to $1 \times 10^{9} / \mathrm{mL}$ in phosphate buffer containing $2 \%$ sodium carboxy methylcellulose (CMC) (Macklin, Shanghai, China).

Fifteen mice were randomly divided into three groups: Sham, P.g+ATRA, and P.g+CMC ( $\mathrm{n}=5$ mice/group). Periodontitis model was induced in mice by oral infection with $10^{9} \mathrm{P}$. gingivalis W83, suspended in $100 \mu \mathrm{l}$ sterile phosphate-buffered saline (PBS) containing $2 \%$ CMC, twice a day for 7 days. Sham mice were 
administered $100 \mu \mathrm{l}$ of PBS containing $2 \% \mathrm{CMC}$ by a micropipette for 7 days. Mice in the P.g+ATRA group were intra-gastrically administered $200 \mu \mathrm{L}$ of ATRA per mouse dissolved in $1 \% \mathrm{CMC}$ solution at $0.25 \mathrm{mg} /$ $\mu \mathrm{l}$ every other day from day 0 to day 42 , and mice in the $P . g+C M C$ and Sham group were intra-gastrically administered $200 \mu \mathrm{l}$ of $1 \% \mathrm{CMC}$ solution per mouse. All mice were killed by asphyxiation with $\mathrm{CO}_{2}$ on day 42 and subjected to subsequent analyses described below.

\section{Bone loss measurement}

The right maxilla was dissected out and the soft tissue was removed by soaking in $3 \%$ hydrogen peroxide for $6 \mathrm{~h}$, followed by boiling for $5 \mathrm{~min}$ to remove the residual soft tissue; the tissues were then stained with $0.1 \%$ methylene blue. The distance from the cement enamel junction (CEJ) of the maxillary first molar to the alveolar bone crest (ABC) of the third molar (CEJ-ABC distance) was measured under a stereomicroscope at $32 \times$ magnification. The mesial as well as the buccal and palatal molar surfaces were measured in each tooth, and the average values of 12 sites were determined. The total CEJ-ABC distance of the 12 sites in each group was calculated. The bone resorption rate of the experimental group (\%) was calculated as $=$ (total CEJ-ABC distance of the experimental group - total CEJ-ABC distance of the Sham group) / total CEJ-ABC distance of the Sham group.

\section{Histological examination}

Staining with hematoxylin and eosin (H\&E) was performed to visualize the pathological damage of periodontal tissue. The molar segment of the left maxilla was washed with phosphate buffer, fixed with $4 \%$ paraformaldehyde for $48 \mathrm{~h}$, and decalcified in 10\% neutral ethylenediamine tetra acetic acid (EDTA; Solarbio). After dehydration in an alcohol series and paraffin embedding, the samples were cut into proximal and distal sections ( $5 \mu \mathrm{m}$ thick). The sections were then stained with H\&E and examined under a dissecting microscope at $100 \times$ magnification.

\section{Flow cytometric analysis}

\section{Isolation of gingival mononuclear cells}

The isolated periodontal tissue was washed with PBS and then added to $1 \mathrm{~mL}$ of PBS containing $2 \%$ FCS, $2 \mathrm{mg} / \mathrm{mL}$ collagenase II (Invitrogen, CA, USA), $1 \mathrm{mg} / \mathrm{mL}$ DNase I (Solarbio), and $1 \mathrm{mg} / \mathrm{mL}$ hyaluronidase (Sigma-Aldrich) in centrifuge tubes. Next, $20 \mu \mathrm{L}$ of $0.5 \mathrm{M}$ EDTA was added, and the tubes were incubated horizontally in a water bath preheated at $37^{\circ} \mathrm{C}$ with shaking at $200 \mathrm{rpm}$ for $60 \mathrm{~min}$. After centrifugation at $400 \mathrm{xg}, 4^{\circ} \mathrm{C}$ for $8 \mathrm{~min}$, the supernatant was discarded and the pellet was passed through a 200 mesh sieve. The filtered cell suspension was then centrifuged at $320 \mathrm{xg}, 4^{\circ} \mathrm{C}$ for $8 \mathrm{~min}$, and the supernatant was 
discarded. The pellet was resuspended in $200 \mu \mathrm{L}$ of $2 \%$ fetal bovine serum (FBS) in PBS (FBS-PBS) and the cell numbers were counted.

\section{Extraction of peritoneal lavage fluid}

Under aseptic conditions, the abdominal cavity of mice was opened and injected with $5 \mathrm{~mL}$ of precooled $2 \%$ FBS-PBS gently over a period of 2-3 min. After holding for an additional $5 \mathrm{~min}$, the peritoneal lavage fluid was absorbed and lavaged again. The fluid was collected into $15-\mathrm{mL}$ centrifuge tubes and the tubes were centrifuged at $300 \times g, 4^{\circ} \mathrm{C}$ for $8 \mathrm{~min}$. The supernatant was discarded; the pellet was resuspended in $200 \mu \mathrm{L}$ of $2 \%$ FBS-PBS and the cell numbers were counted.

\section{Preparation of single cell suspensions of spleen cells}

The spleens of mice were removed aseptically according to routine procedures, rinsed with $1 \mathrm{~mL}$ of PBS, ground on a 200-mesh stainless steel screen, and filtered through a 30- $\mu \mathrm{m}$ nylon mesh to remove agglomerated cells. The cell suspension was centrifuged at $300 \mathrm{xg}, 4^{\circ} \mathrm{C}$ for $5 \mathrm{~min}$, and then the supernatant was aspirated. Next, $1 \mathrm{~mL}$ red blood cell lysate was added to the precipitated cell mass. RPMI1640 medium containing 10\% FBS and 1\% penicillin-streptomycin was then added to terminate the reaction. After centrifugation at $300 \mathrm{xg}, 4^{\circ} \mathrm{C}$ for $5 \mathrm{~min}$, the supernatant was discarded. The pellet was then washed twice with PBS and the supernatant was discarded. Cell viability was determined by trypan blue staining, and the cells were counted.

To identify macrophages and macrophage subpopulations, cells were stained with FITC-labeled monoclonal anti-mouse F4/80 and APC-labeled monoclonal anti-mouse CD206 antibodies (BioLegend, San Diego, CA, USA). The cells were washed twice with PBS containing $1 \% \mathrm{FBS}$, resuspended in staining buffer, and examined by flow cytometry (BD FACSAria II, NY, USA). F4/80 ${ }^{+} \mathrm{CD}_{206}{ }^{+}$cells (M2 macrophages) were analyzed using Flow Jo10.4 software.

\section{Quantitative reverse transcription-polymerase chain reaction (qRT-PCR)}

For qRT-PCR, total RNA was extracted from gingival tissues, peritoneal cells, and splenocytes using TRIzol reagent (Vazyme Biotechnology Co. Ltd, Nanjing, China) in accordance with the manufacturer's protocol. The quality and quantity of RNA were measured using a spectrophotometer. The absorbance was measured at wavelengths of $260 \mathrm{~nm}$ and $280 \mathrm{~nm}$ and the ratios of the optical densities (ODs) at 260/280 $\mathrm{nm}$ for the samples were between 2.0 and 2.2. Total RNA was reverse transcribed into CDNA for amplification using reverse transcription (RT) reaction (PrimeScript RT system; Vazyme Biotechnology Co. Ltd.) according to the manufacturer's instructions. PCR was performed in an ABIPRISM cycler with the SYBR Green PCR Kit and specific primers for IL-10, Arg-1, TGF-b1, and b-actin mRNAs. PCR was 
performed in a total volume of $20 \mu \mathrm{L}$ consisting of $10 \mu \mathrm{L}$ of $2 \times$ ChamQuniversal SYBR qPCR Master Mix, $0.4 \mu \mathrm{L}$ each of forward and reverse primers, $2 \mu \mathrm{L}$ of $\mathrm{cDNA}$, and $\mathrm{ddH}_{2} \mathrm{O}$.

The primer sequences are as follows:

mouse IL-10, forward, 5'-CCTCGTTTGTACCTCTCTCCG-3';

mouse IL-10, reverse, 5'-AGGACACCATAGCAAAGGGC-3';

mouse TGF-b1, forward, 5'-AGCTGCGCTTGCAGAGATTA-3';

mouse TGF-b1, reverse, 5'-AGCCCTGTATTCCGTCTCCT-3';

mouse Arg-1, forward, 5'-CAGCACTGAGGAAAGCTGGT-3';

mouse Arg-1, reverse, 5'-ACAGACCGTGGGTTCTTCAC-3';

mouse b-actin, forward, 5'-GGCTGTATTCCCCTCCATCG-3';

mouse b-actin, reverse, 5'-CCAGTTGGTAACAATGCCATGT-3'.

The $2^{-\Delta \Delta C t}$ method was used to determine the relative expression level of each gene. The target gene mRNA expression was normalized relative to b-actin mRNA, and the adjusted gene expression of mice in the Sham group was used as a reference (fold change $=1$ ).

\section{Statistical analysis}

The data are expressed as the mean \pm standard deviation (SD) and were analyzed using GraphPad Prism 22.0 software. The results were analyzed by one-way analysis of variance (ANOVA) and Student's $t$ test. In all analyses, $P<0.05$ was taken to indicate statistical significance.

\section{Results}

\section{ATRA prevented the body weight loss in mice induced by $P$. gingivalis infection}

To examine the effects of ATRA on the mouse model of periodontitis established by infection with $P$. gingivalis, we first compared the changes in body weight between the P.g+ATRA, P.g+CMC, and Sham groups. The mice in the $P . g+C M C$ group weighed significantly less than those in the Sham group on day $21(P<0.01$,Figure 1). The body weights of the mice in the P.g+ATRA group began to show a significant upward trend on day 21 compared with mice in the $P . g+C M C$ group, which continued until the animals were sacrificed on day $42(P<0.01$,Figure 1$)$. These data suggest that ATRA can improve the changes in body weight in periodontitis mice, possibly by ameliorating the effects of periodontitis. 


\section{ATRA reduced alveolar bone loss induced by $P$. gingivalis infection}

To examine the effects of ATRA on periodontitis in mice infected with $P$. gingivalis, we measured the CEJABC distance in the P.g+ATRA, P.g+CMC, and Sham groups. The CEJ-ABC distance was significantly increased in the $P . g+C M C$ mice compared with the distance in the Sham mice $(P<0.01$, Figure $2 a, b)$. However, the CEJ-ABC distance in P.g+ATRA mice was greatly reduced compared with the distance in the $P . g+C M C$ mice $(P<0.01)$. No significant difference was observed in alveolar bone resorption between the $P . g+$ ATRA mice and Sham mice $(P<0.05$, Figure $2 b)$.

The alveolar bone resorption rates of both the Sham and the P.g+ATRA mice were significantly lower than that of the $P . g+C M C$ mice $(0 \% \pm 6.6 \%$ and $16.2 \% \pm 7.4 \%$ vs. $54.3 \% \pm 8.7 \%$, respectively; all, $P<0.01$, Figure 2c).

\section{ATRA attenuated periodontal lesions induced by $P$. gingivalis}

Histological analysis with H\&E staining indicated that the epithelium in the sulcus was intact in the Sham mice; there was no inflammatory cell infiltration in gingival epithelium and the periodontal ligament fiber showed an orderly and regular arrangement (Figure 3). In contrast, the P.g+CMC mice showed inflammatory cell infiltration and collagen fiber degeneration and destruction. We also detected infiltration of large numbers of inflammatory cells and loss of attachment. ATRA treatment resulted in reduced inflammatory cell infiltration and attachment losses in $P$. gingivalis-infected periodontal tissues. Moreover, there was no apparent alveolar bone resorption in P.g+ATRA mice compared with that observed in $P . g+C M C$ mice.

\section{ATRA increased the proportion of M2 macrophages during $P$. gingivalis infection}

To clarify the role of ATRA in the immune status of M2 macrophages in mouse periodontitis, the percentages of $\mathrm{F} 4 / 80^{+} \mathrm{CD} 206^{+}$cells (M2 macrophages) in gingivae, peritoneal cavity, and spleen were analyzed by flow cytometry. The results showed that the proportions of $\mathrm{F} 4 / 80^{+} \mathrm{CD} 206^{+} \mathrm{M} 2$ macrophages in gingival mononuclear cells, peritoneal lavage fluid (PLF), and spleen were significantly decreased in the $P . g+\mathrm{CMC}$ mice compared with levels in the Sham mice $(P<0.01$, Figure 4$)$. In contrast, the P.g+ATRA mice showed significantly higher proportions of $\mathrm{F} 4 / 80^{+} \mathrm{CD} 206^{+} \mathrm{M} 2$ macrophages in gingival mononuclear cells, PLF, and spleen compared with levels in the $P . g+\mathrm{CMC}$ mice $(P<0.01$, Figure 4$)$. 


\section{ATRA upregulated the mRNA expression of M2 macrophage-related cytokines in periodontitis}

qRT-PCR analyses were performed to determine the mRNA expression levels of M2 macrophage-related cytokines in gingivae, PLF, and spleen. The levels of IL-10 and TGF-b1 mRNAs in gingivae and PLF were significantly increased in the $P . g+C M C$ mice compared with levels in the Sham mice $(P<$ 0.01 , Figure $5 a, b)$. Arg1 mRNA expression levels in gingival tissue were significantly higher in the $P . g+C M C$ mice than in the Sham mice $(P<0.01$, Figure $5 a)$, but there were no significant differences in the PLF and spleen ( $P>0.05$, Figure 5b, $c)$. The levels of IL-10, TGF-b1, and Arg-1 mRNAs in the gingivae, PLF, and spleen were significantly higher in the $P . g+A T R A$ mice than in the $P . g+C M C$ mice $(P<0.01$, Figure 5ac).

\section{Discussion}

The clinical treatment of periodontitis currently includes plaque removal, surgical treatment, auxiliary laser, drug therapy, and other treatments. However, plaque removal and periodontal surgery have limited efficacy in the management of periodontitis [12]. Given the critical role of the immune system in the occurrence, development, and prognosis of periodontitis, effective regulation of the immune response is critical to enable it to play a protective role against periodontitis. Macrophages are involved in the development and persistence of inflammatory responses and the protection against periodontitis [13]. M2 macrophages are immunomodulatory cells that are characterized by the expressions of c-MAF, Arginase1, CD14, CD163, mannose receptor (CD206), IL-10 and TGF- $\beta 1$ [14]. Our study results indicate that ATRA upregulated the immune status of $\mathrm{M} 2$ macrophages in a mouse model of periodontitis and effectively inhibited periodontal tissue destruction. ATRA increased the proportions of $F 4 / 80^{+} C D 206^{+} \mathrm{M} 2$ macrophages in gingival tissue, spleen, and PLF and upregulated the expression of IL-10, TGF-b1, and Arg-1 mRNAs, which may have a protective effect against periodontitis.

Some studies have shown that $P$. gingivalis inhibits the production of a-ketoglutaric acid by M2 macrophages, thus maintaining the high inflammatory state of the periodontal microenvironment [15]. Miao et al.[16] reported that the proportion of M2 macrophages was significantly smaller in a mouse model of periodontitis induced by $P$. gingivalis compared with the healthy control group. These results imply that M2 macrophages are involved in maintaining periodontal tissue homeostasis in the periodontal microenvironment. Consistent with the above results, we found that the proportion of M2 macrophages in the gingival tissue of mice with periodontitis decreased in the model mice compared with the Sham group, while the mRNA expression levels of M2 macrophage-related cytokines IL-10, TGFb1, and Arg1 increased. Although the levels of anti-inflammatory factors increased in mice with periodontitis, periodontal attachment loss and alveolar crest resorption were observed, which may have been because the efficacy of the anti-inflammatory cytokines secreted by M2 macrophages was insufficient to resist the tissue destruction caused by inflammation with the progression of inflammatory periodontitis. These results indicate a weak macrophage-mediated immune response in periodontitis. 
Fotinoset al.[17] reported that the autoimmune reaction caused by severe periodontitis resulted in the accumulation of large numbers of immune cells, such as macrophages, in the periodontal tissue that destroyed the basement membrane. Destruction of the basement membrane allowed toxic compounds such as lipopolysaccharide to penetrate across the gingival epithelial barrier and enter the bloodstream, resulting in a continuous stimulation of the systemic host response and increased levels of inflammation and C-reactive protein in the body. Consistent with the findings of Fotinos et al., in the present study, we detected the presence of $\mathrm{M} 2$ macrophages and the expression of M2 macrophage-related factors in the PLF and spleen of mice with periodontitis. The results were consistent with the changes in the proportion of M2 macrophages seen in gingival tissue. The proportion of M2 macrophages decreased in the PLF and spleen, but there were no significant changes in anti-inflammatory cytokine levels. These observations imply that the immune response mediated by M2 macrophages in the PLF and spleen is weakened in periodontitis.

Enhancement of the immune response mediated by $\mathrm{M} 2$ macrophages has been reported to ameliorate the inflammatory response and promote tissue repair. Hunter et al. [8] reported that adoptive infusion of M2 macrophages reduced the degree of inflammation in mice with colitis induced by dinitrobenzene sulfonic acid. In an animal model of experimental autoimmune encephalomyelitis, Liu et al. [18] reported that upregulation of the expressions of Arg-1, CD206, CD14, and IL-10 in the spleen induced the transformation of inflammatory M1 macrophages into anti-inflammatory M2 macrophages, thus increasing demyelination and inhibiting the progression of inflammation. Zhuang et al. [19] reported that the proportion of $\mathrm{CD} 206^{+} \mathrm{M} 2$ macrophages in gingival tissue increased, the number of osteoclasts decreased, and bone resorption decreased following injection of CCL2 into the gingivae of mice orally infected with $P$. gingivalis.

ATRA has a number of biological roles in cell proliferation and differentiation, embryogenesis, immunity, and metabolism [20]. ATRA was reported to induce the secretion of M2 macrophage chemokines in both C57BL/6 (B6) and BALB/c mice by inhibiting the expression of pro-inflammatory cytokines, nitric oxide synthase (NOS2), and NO and promoted the transition of macrophages from M1 to M2 in the two mouse strains [10]. Feng et al.[3] reported that ATRA reduced the expression of CD80, CD86 and CCR7 in splenic macrophages, increased the expression of CD209, promoted the polarization of macrophages to M2 macrophages, enhanced the production of IL-10 in M2 macrophages and inhibited the secretion of IL-12 and TNF-a, which further alleviated the progression of idiopathic thrombocytopenic purpura. Our previous studies confirmed that ATRA regulates the imbalance of Th17/Treg cells and improves periodontitis caused by $P$. gingivalis. These activities were likely mediated by ATRA inhibiting the response of Th17 cells, enhancing the response of Treg cells, and reducing the expression of RANKL in CD $4^{+} \mathrm{T}$ cells, thus regulating the Th17/Treg imbalance[21]. In the present study, oral administration of ATRA in a $P$. gingivalis-induced periodontitis mouse model resulted in an increase in $\mathrm{F} 4 / 80^{+} \mathrm{CD} 206^{+} \mathrm{M} 2$ macrophages; there was no obvious alveolar bone resorption, and the mRNA expression levels of M2 macrophage-related factors IL-10, TGF-b1, and Arg1 in gingival tissue increased. Similar results were 
observed in the spleen and PLF. Therefore, ATRA not only modulates the immune state of M2 macrophages in the periodontal region, but also regulates the systemic immune state.

ATRA has been reported to activate JAK1/3 by regulating the binding of IL-4 with its receptor IL-4R. Phosphorylated JAK1/3 then recruits signal transduction and transcriptional activator 6 (STAT6) to promote the gene expression of Arg1, a key gene for tissue repair in M2 macrophages [22]. Lee et al. [23] reported that ATRA activates Arg-1 by upregulating the transcription of biosynthetic rate-limiting enzyme Raldh2, thereby promoting wound healing. ATRA was reported to induce the expression of TIMP1 in human alveolar M2 macrophages and regulate tissue remodeling by inhibiting the degradation of the extracellular matrix by various matrix metalloproteinases[24] . Retinoic acid nuclear receptor RAR-b is highly expressed in peritoneal macrophages, and retinoic acid response elements are also present in the gene regulatory regions of specific GATA6 transcription factors expressed in peritoneal macrophages. ATRA regulates the expression of Arg-1-specific genes in peritoneal macrophages through GATA6 transcription factors $[25,26]$. Taken together, the above results imply that ATRA affects the polarization of macrophages through a variety of signaling pathways.

In conclusion, the results of this study demonstrate that oral administration of ATRA modulated the immune status of M2 macrophages, affected the local oral microenvironment, and improved the inflammatory state in an experimental mouse model of P. gingivalis-induced periodontitis. Treatment with ATRA reduced both alveolar bone loss and the systemic immune response. These observations provide a basis for studying the influence of periodontitis on innate immune function, as well as the relationships between periodontitis, systemic health, and immune regulation of periodontitis.

\section{Declarations}

\section{Acknowledgements}

The authors thank Dr. Yaping Pan of the Department of Periodontics, School of Stomatology, China Medical University, Shenyang, China, for kindly providing P. gingivalis W83.

\section{Funding}

This work was supported by the National Natural Science Foundation of China (GrantNO.81600870).

\section{Conflict of Interest statement}

The authors declare that they have no competing interests.

\section{Ethics approval and consent to participate}

The present study was approved by the Ethical Committee of Jinzhou Medical University .

\section{Authors' contributions}


WJZ and LYW conceived the study. WJZ drafted the manuscript. LYW revised the manuscript. Flow cytometry was completed by WLY. P.gingivalis W83 culture and mouse periodontitis model was completed by XQG and NG. Reverese transcription- quantitative PCR was performed by WJZ. All authors have read and approved the manuscript.

Consent to participate All authors listed in the article have approved the manuscript that is enclosed.

Consent to publish The manuscript is approved by all authors for publication.

Informed consent All authors agree and give consent for the publication.

\section{References}

1. Cardoso EM, Reis C, Manzanares-Cespedes MC(2017) Chronic periodontitis, inflammatory cytokines, and interrelationship with other chronic diseases. Postgrad Med 130(1):98-104. https://doi.org/10.1080/00325481.2018.1396876

2. Seymour GJ, Gemmell E, Reinhardt RA, Eastcott J et al (2010) Immunopathogenesis of chronic inflammatory periodontal disease: cellular and molecular mechanisms. J Periodontal Res 28(6):478486. https://doi.org/10.1111/j.1600-0765.1993.tb02108.x

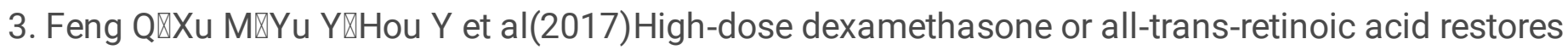
the balance of macrophages towards M2 in immune thrombocytopenia. J Thromb Haemost 15:1845-1858. https://doi.org/10.1111/jth.13767

4. Wynn TA, Barron L, Thompson RW, Madala SK et al(2001) Quantitative assessment of macrophage functions in repair and fibrosis. Current Protocols in Immunology 93(1):1-15. https://doi.org/10.1002/0471142735.im1422s93

5. Ckless K, Lampert A, Reiss J, Kasahara D et al(2008) Inhibition of arginase activity enhances inflammation in mice with allergic airway disease, in association with increases in protein snitrosylation and tyrosine nitration. JJ Clin Immunol 181(6): 4255-64. https://doi.org/10.4049/jimmunol.181.6.4255

6. Mantovani A, Sozzani S, Locati M, Allavena et al(2002)Macrophage polarization: tumor-associated macrophages as a paradigm for polarized M2 mononuclear phagocytes. Trends Immunol 23(11): 549-555.https://doi.org/

7. Bi Y, Chen J, Hu F, Liu J et al(2019) M2 Macrophages as a Potential Target for Antiatherosclerosis Treatment.Neural Plast 2019: 1-21.https://doi.org/10.1155/2019/6724903

8. Hunter MM, Wang A, Parhar KS, Johnston M et al(2010) In vitro-derived alternatively activated macrophages reduce colonic inflammation in mice. Gastroenterology 138(4):13951405.https://doi.org/10.1053/j.gastro.2009.12.041

9. Zhan X, Liu Y, Yang J, Wang G et al(2012) All- trans -retinoic acid ameliorates experimental allergic encephalomyelitis by affecting dendritic cell and monocyte development. Immunology 138(4). https://doi.org/10.1111/imm.12040 
10. Vellozo NS, Pereira-Marques ST, Cabral-Piccin MP, Filardy AA et al (2017) All-trans retinoic acid promotes an $\mathrm{m} 1$-to m2-phenotype shift and inhibits macrophage-mediated immunity to leishmania major. Front Immunol 8:1560. https://doi.org/10.3389/fimmu.2017.01560

11. Surace MJ, Li L(2013)Potent suppression of arginase 1 expression in murine macrophages by low dose endotoxin. Am J Clin Exp Immunol 2(1): 117-123.

12. Sanz M, Herrera D, Kebschull M, Chapple I et al(2020) Treatment of stage I-III periodontitis-The EFP S3 level clinical practice guideline. J Clin Periodontol 47 Suppl 22(Suppl 22):4-60. https://doi.org/10.1111/jcpe.13290

13. Sima C, Glogauer M(2012)Macrophage subsets and osteoimmunology: tuning of the immunological recognition and effector systems that maintain alveolar bone. Periodontology 63(1): 80-101. https://doi.org/10.1111/prd.12032

14. Barboza TC, Sotto MN, Kanashiro-Galo L, de Brito AC, et al(2020) M2-Polarized Macrophages Determine Human Cutaneous Lesions in Lacaziosis. Mycopathologia 185(3):477-483. https://doi.org/10.1007/s11046-020-00450-z

15. Yu S, Ding L, Liang D, Luo L(2018)Porphyromonasgingivalis inhibits $\mathrm{m} 2$ activation of macrophages by suppressing a-ketoglutarate production in mice. Mol Oral Microbiol 33(5). https://doi.org/10.1111/omi.12241

16. Miao Y, He L, Qi X, Lin X(2020)Injecting Immunosuppressive M2 Macrophages Alleviates the Symptoms of Periodontitis in Mice. Front Mol Biosci. 7:1-10. https://doi.org/10.3389/fmolb.2020.603817

17. Fotinos S Panagakos(2012)Periodontal disease and systemic health. Dental Nursing 8(3):130-135区 https://doi.org/CliCa0103315318

18. Liu C, Li Y, Yu J, Feng L et al(2013) Targeting the shift from M1 to M2 macrophages in experimental autoimmune encephalomyelitis mice treated with fasudil. Plos One 8(2): e54841. https://doi.org/10.1371/journal.pone.0054841

19. Zhuang Z, Yoshizawa-Smith S, Glowacki A, Maltos K et al(2019) Induction of M2 Macrophages Prevents Bone Loss in Murine Periodontitis Models. J Dent Res 98(2):200-208. https://doi.org/10.1177/0022034518805984

20. Al Tanoury Z, Piskunov A, Rochette-Egly C(2013) Vitamin a and retinoid signaling: genomic and nongenomic effects. J Dent Res 54(7): 1761-1775. https://doi.org/10.1194/jlr.R030833

21. Wang L, Wang J, Jin Y, Gao H et al(2014) Oral Administration of All-Trans Retinoic Acid Suppresses Experimental Periodontitis by Modulating the Th17/Treg Imbalance. J Dent Res 85(5):740-750. https://doi.org/10.1902/jop.2013.130132

22. Mikita T, Campbell D, Wu P, Williamson K et al(1996) Requirements for interleukin-4-induced gene expression and functional characterization of Stat6. J Dent Res 16(10): 5811-5820. https://doi.org/10.1128/MCB.16.10.5811

23. Lee B, Wu CY, Lin YW, Park SW et al(2016) Synergistic activation of arg1 gene by retinoic acid and il4 involves chromatin remodeling for transcription initiation and elongation coupling. Nucleic Acids 
Res 44(16): 7568-7579. https://doi.org/10.1093/nar/gkw392

24. Csóka B, Selmeczy Z, Koscsó B, Németh ZH et al(2012) Adenosine promotes alternative macrophage activation via a2a and a2b receptors. Faseb J 26: 376-386. https://doi.org/10.1096/fj.11-190934

25. Okabe Y, Medzhitov R( 2014) Tissue-specific signals control reversible program of localization and functional polarization of macrophages. Cell 157(4):832-844.

https://doi.org/10.1016/j.cell.2014.04.016

26. Zhu B, Buttrick T, Bassil R, Zhu C et al(2013) Il-4 and retinoic acid synergistically induce regulatory dendritic cells expressing aldh1a2. J Clin Immunol 191(6):3139-

3151.https://doi.org/10.4049/jimmunol.1300329

\section{Figures}

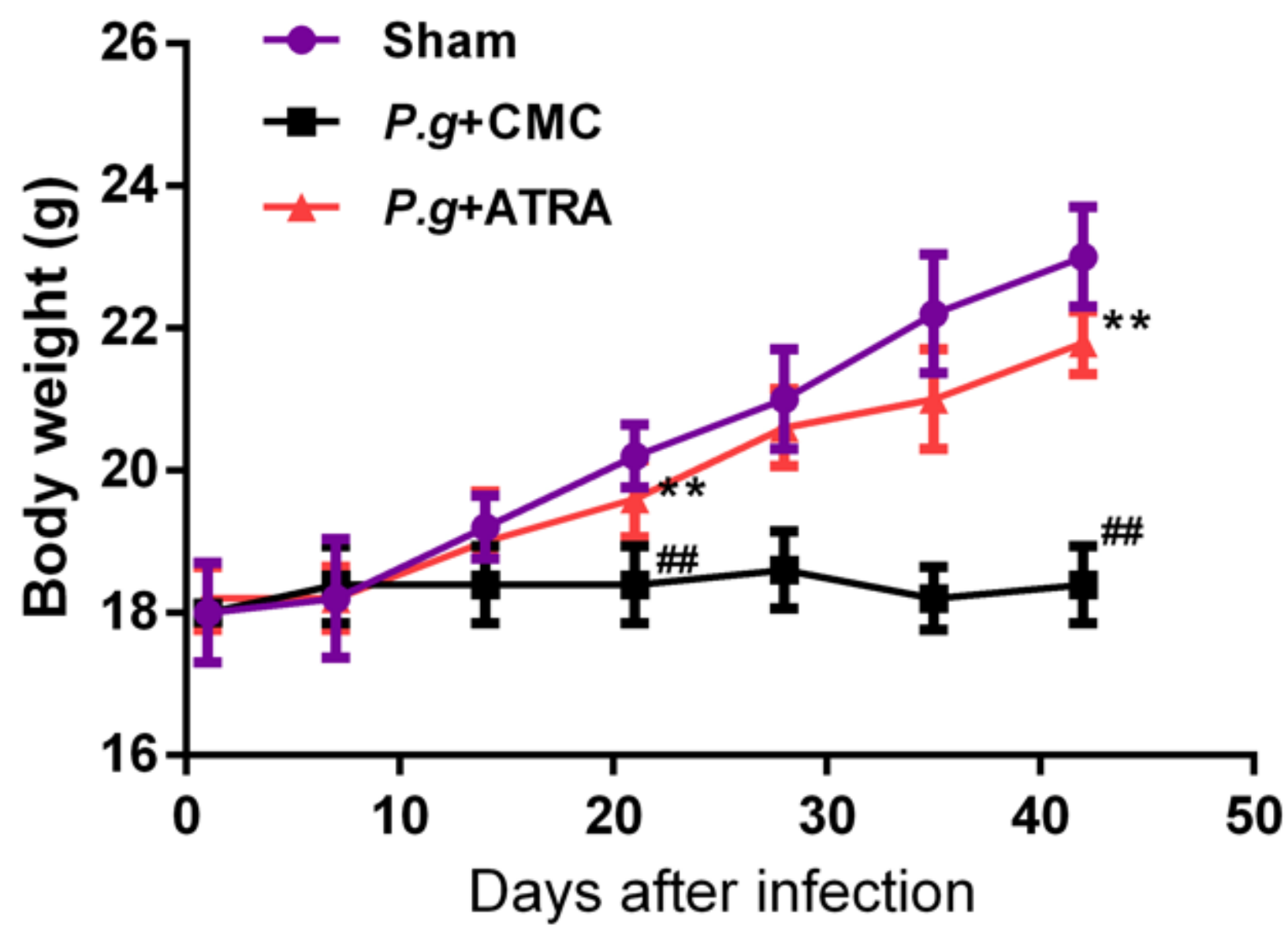

Figure 1

ATRA improved the body weight changes in periodontitis mice. Note: \#\# $\mathrm{P}<0.01$ vs. Sham group; $* \star \mathrm{P}<$ 0.01 vs. P.g+CMC group. 
a
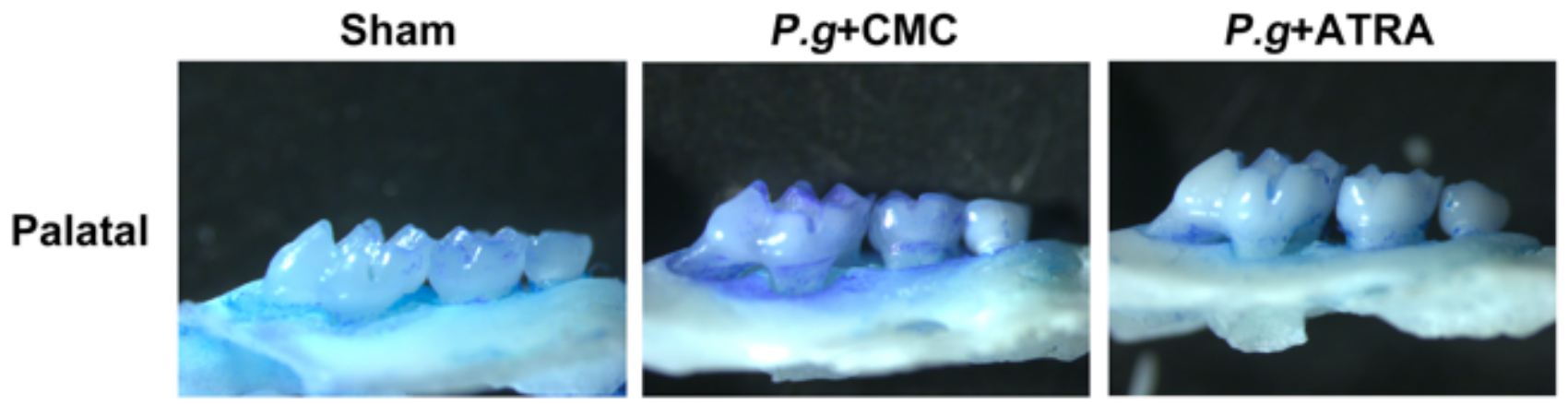

Buccal
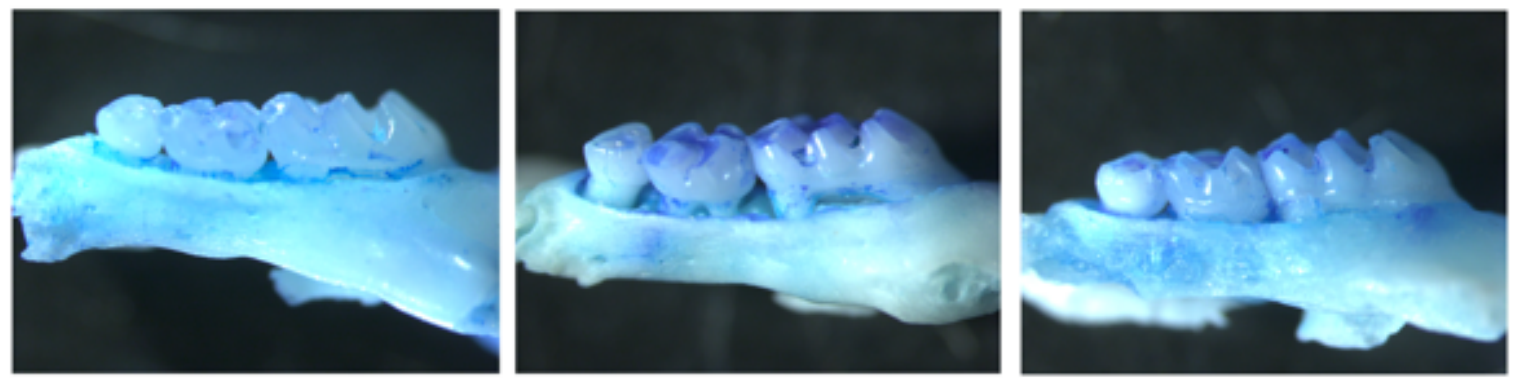

b

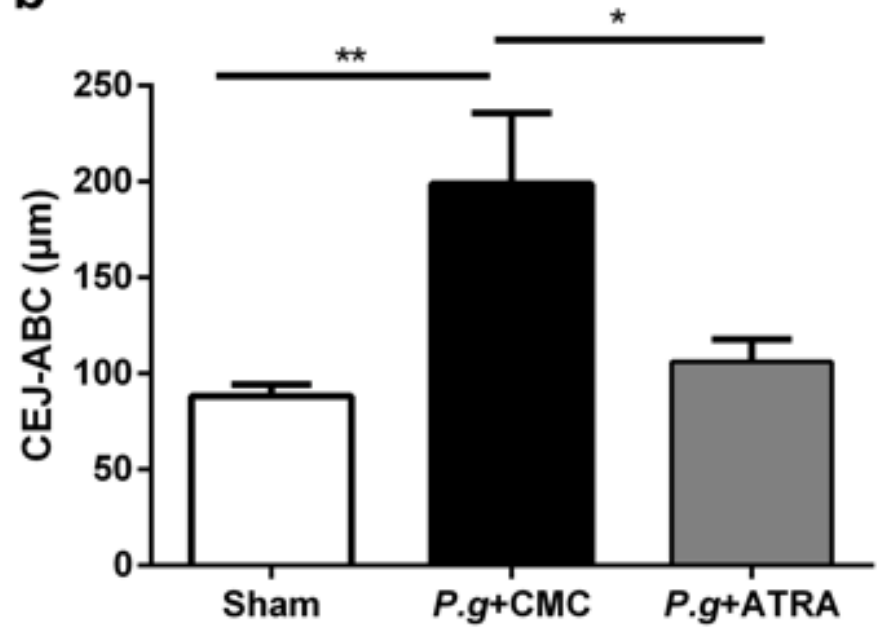

C

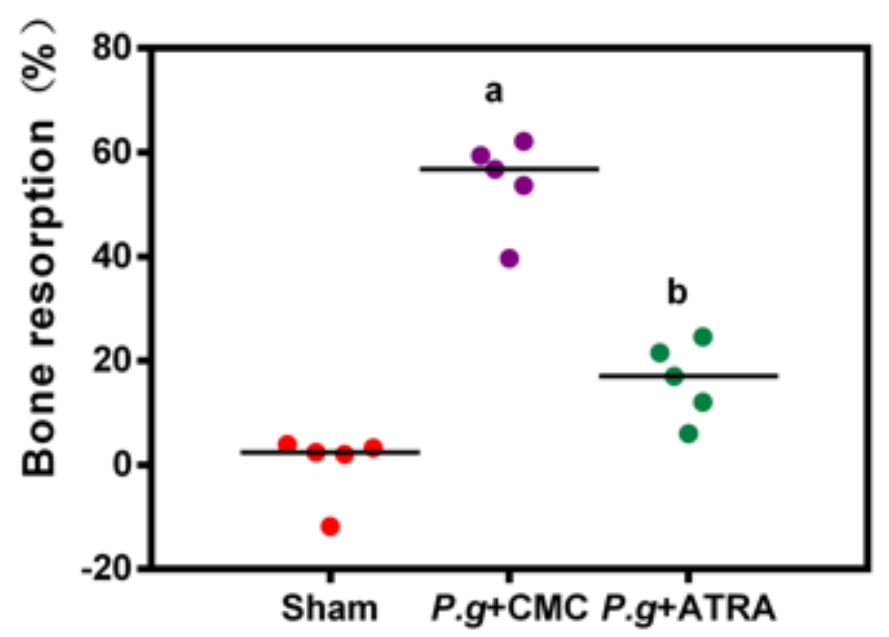

Figure 2

Evaluation of alveolar bone resorption in experimental periodontitis. $(a, b)$ Alveolar bone resorption was analyzed by measuring the distance from the CEJ to the $A B C$ on both the buccal and palatal molar surfaces. Representative images are shown (32x magnification) (a). Values are shown as the mean \pm SD of three mice per group. ${ }^{\star} P<0.05,{ }^{\star} \mathrm{P}<0.01$ (b). (c) Alveolar bone resorption rate. a: $\mathrm{P}<0.01$ vs. Sham group; b: $\mathrm{P}<0.01$ vs. $\mathrm{P.g}+\mathrm{CMC}$ group. 
Sham

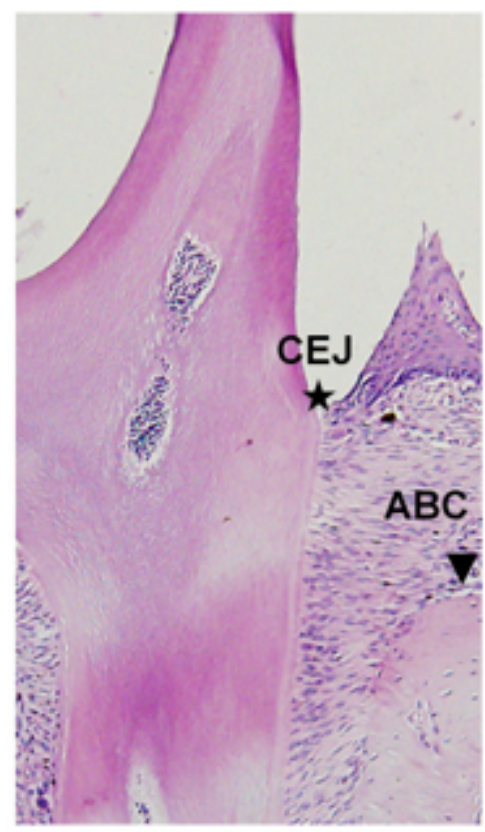

P.g+CMC

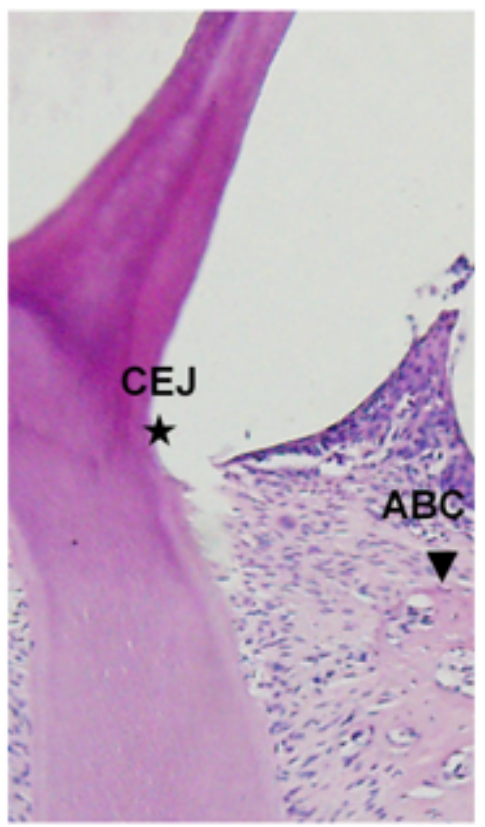

P.g+ATRA

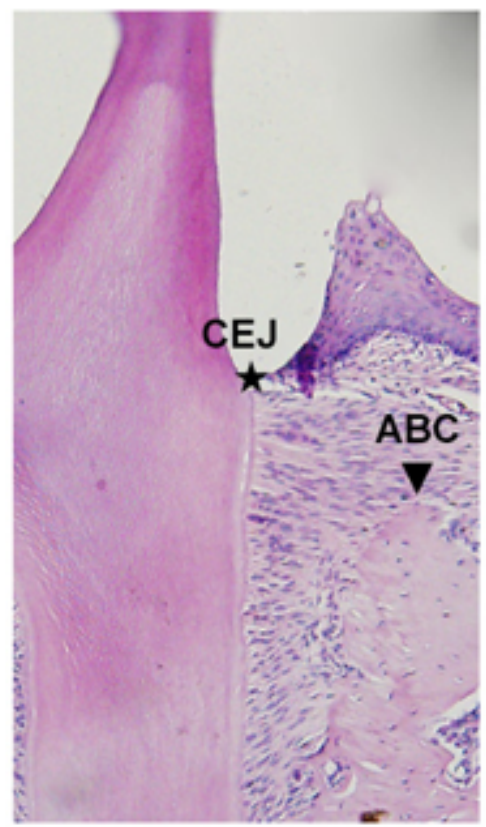

\section{Figure 3}

Histological examination of periodontal tissues (100x magnification). ABC, alveolar bone crest; CEJ; cementoenamel junction. 


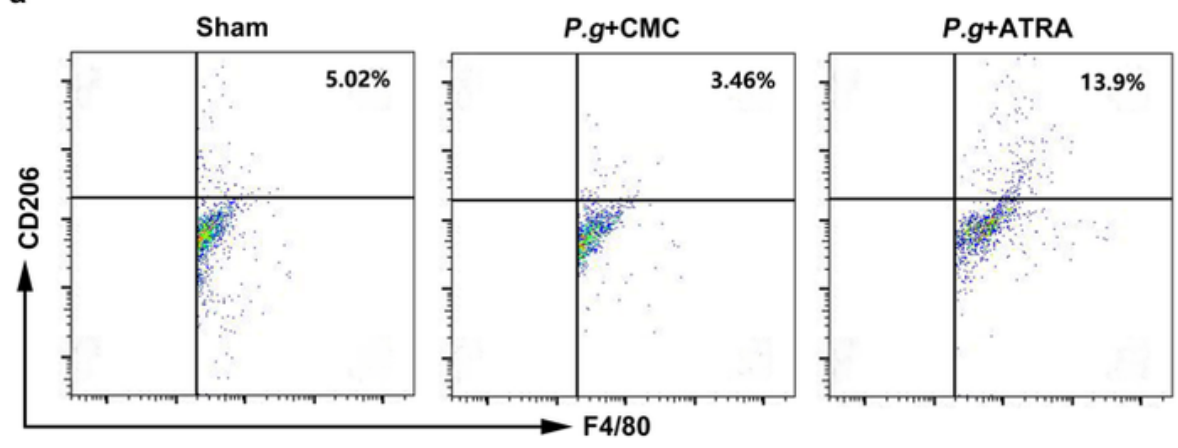

b
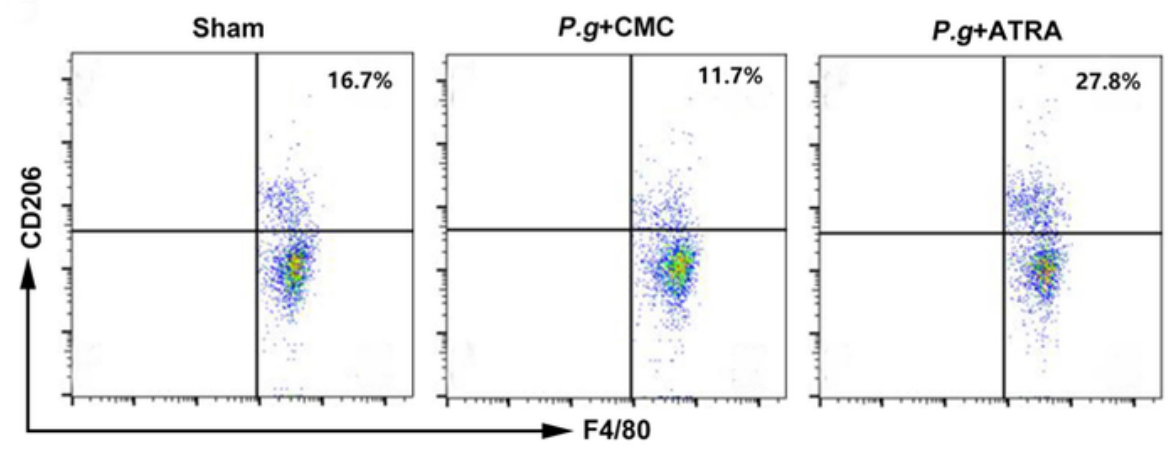

C
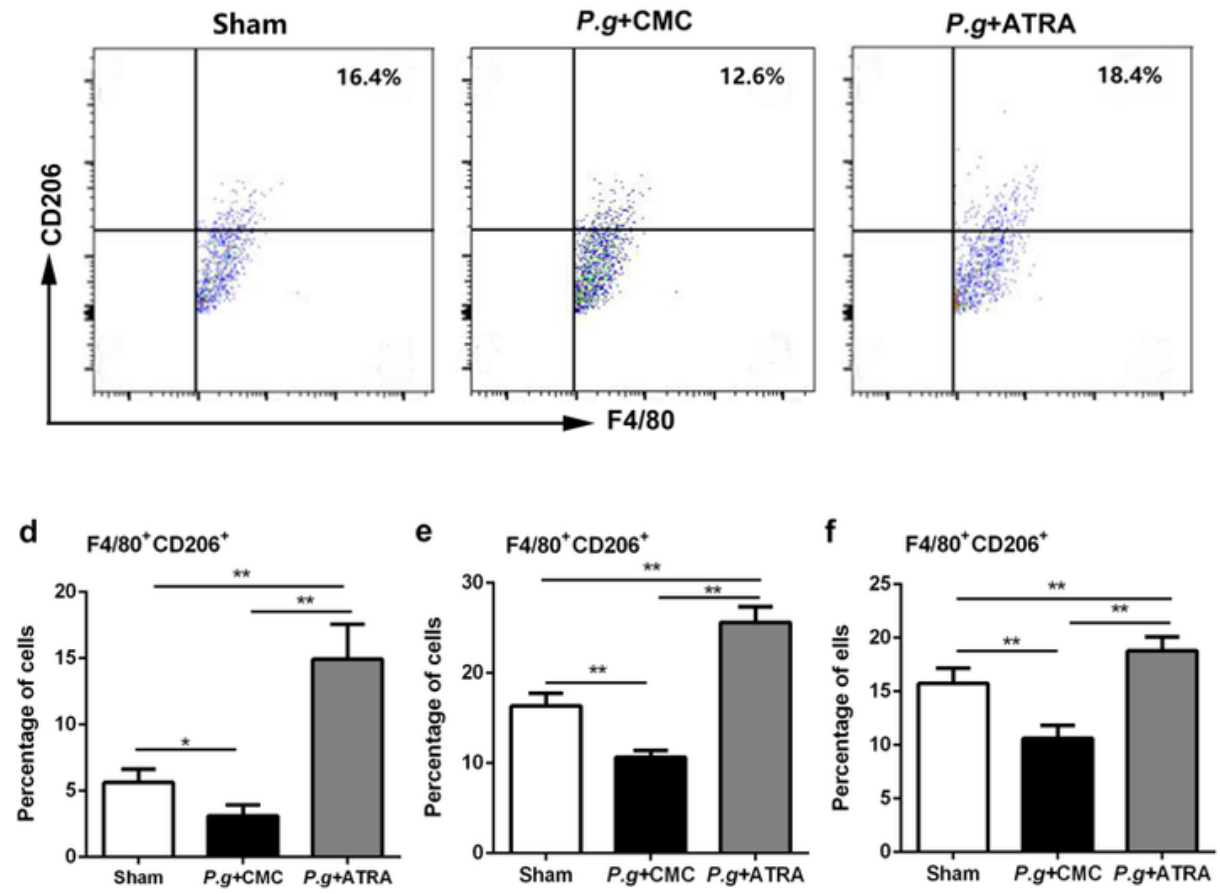

\section{Figure 4}

Oral administration of ATRA increased the proportion of M2 macrophages in P. gingivalis-infected mice. Flow cytometric analysis of M2 macrophages. Representative dot plots for F4/80+CD206+ (M2 macrophages) staining in (a) GMCs, (b) PLF, and (c) spleen. (d, e, f) Percentages of F4/80+CD206+ (M2 macrophages) in GMCs, PLF, and spleen, respectively. Data are shown as the means \pm SD of five mice per 
group. ${ }^{*} \mathrm{P}<0.05,{ }^{\star} \mathrm{P}<0.01$ between the two groups. GMCs, gingival mononuclear cells; PLF, peritoneal lavage fluid.
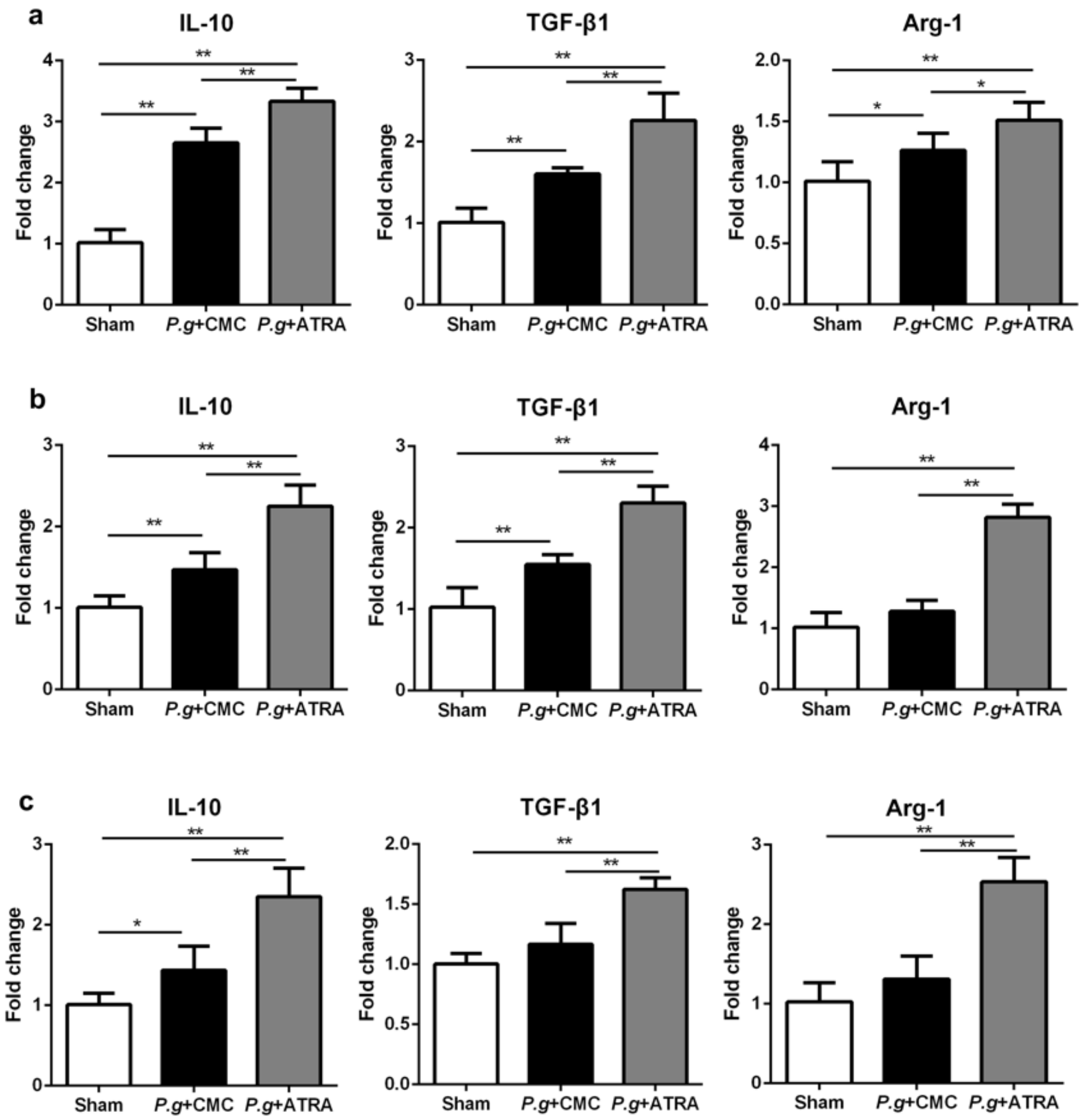

Figure 5

ATRA increased the mRNA expression of M2 macrophage-related cytokines in periodontitis. IL-10, TGF$\beta 1$, and Arg-1 mRNA expressions were determined by qRT-PCR and expressed as fold changes with 
respect to levels in Sham mice (set as 1). (a-c) Fold changes (mean \pm SD) of IL-10, TGF- $\beta 1$, and Arg-1 mRNA in gingivae (A), PLF (b), and spleen (c). ${ }^{*} P<0.05,{ }^{\star *} P<0.01$. 